\title{
Superresolving scanning optical microscopy using holographic optical processing
}

\author{
J. G. Walker, E. R. Pike, R. E. Davies, and M. R. Young* \\ Department of Physics, King's College London, Strand, London WC2R 2LS, UK \\ G. J. Brakenhoff \\ Department of Molecular Biology and Electron Microscopy, University of Amsterdam, \\ Amsterdam, The Netherlands
}

M. Bertero

Department of Physics, University of Genoa, Genoa, Italy

\begin{abstract}
Received October 4, 1990; accepted July 15, 1992; revised manuscript received August 14, 1992
Two novel superrresolving scanning microscopes, one of which uses coherent imaging and the other incoherent imaging, are described. The optical arrangement used in the coherent microscope is similar to that in a scanning confocal microscope with the detector pinhole replaced by a special holographic mask, a Fourier lens, and a pinhole. The incoherent design uses two intensity-transmittance masks, two integrating detectors, and an electronic subtractor. The design of the microscopes is based on the results of singular-system theory, and the mask forms are calculated by means of this analysis. These arrangements obviate the need for an array of detectors to implement singular-system processing, and in the coherent case direct phase measurement is no longer required. Experimental results are presented that demonstrate a significant resolution improvement for a one-dimensional low-numerical-aperture coherent microscope.
\end{abstract}

\section{INTRODUCTION}

Confocal scanning microscopy ${ }^{1,2}$ is now a well-established technique for achieving an enhancement in resolution over the resolution of a conventional microscope, when lenses of the same numerical aperture and light of the same wavelength are used. In a confocal scanning system the object is illuminated by a focused beam; the transmitted, reflected, or fluorescent light is then imaged, and the light that passes through a pinhole at the center of the image plane is detected. In a recent series of papers ${ }^{3-6}$ the theory of singular systems has been applied to the case of scanning microscopy. Singularsystem theory is a generalization of the eigenvalue theory that was first applied to the imaging resolution problem by Slepian and Pollak in 1961. ${ }^{7}$ Whereas the eigenvalue approach considers only the distribution of light within the geometrical image of the object, singular-system analysis treats the case in which the light distribution over the whole image plane is available.

In conventional imaging situations only a small fraction of the light falls outside the geometrical image, and eigenvalue and singular-system analyses give similar results, which yield the Rayleigh resolution criterion. However, in a scanning microscope the spreading of the light, as a result of diffraction, outside the geometrical image is significant, and hence singular-system analysis is more appropriate. The results of this type of analysis indicate that, if the light distribution over the whole image plane is detected and suitably processed, then resolution exceeding the Rayleigh criterion by a factor of 2 may be achieved.
A novel microscope based on these principles was proposed in 1982 by Bertero and Pike. ${ }^{3}$ This microscope is similar in operation to a conventional confocal scanning microscope but uses an array of detectors in the image plane in place of a single pinhole and detector. Signals from the detector array are processed to yield the superresolved signal. In 1983 Walker, ${ }^{8}$ using these principles, obtained experimental confirmation of the predicted superresolution at each scanning point. Application of this technique to scanning microscopy was further quantified by Bertero et al.,6,9,10 and experimental confirmation of their theoretical superresolution predictions have been obtained with the use of both incoherent ${ }^{11}$ and coherhent ${ }^{12,13}$ low-numerical-aperture microscopes. The use of multiple detectors has the disadvantage that the detectors or detector elements must be calibrated. In addition, for the coherent case, signals relating to the complex amplitude are required, thus necessitating the use of interferometry or some other method of phase measurement.

Our purpose in this paper is to describe two novel microscopes..$^{14,15}$ that implement singular-system processing without the need either for a multielement detector array or for phase measurement. This implementation is achieved in the coherent case by the performance of predetection optical processing with the use of a special holographic mask, a Fourier lens, a pinhole, and a single detector. In the incoherent case the processing is performed with the use of two intensity-transmittance masks, two integrating detectors, and an electronic subtractor. A brief Fourier-optics analysis of the imaging properties of the microscopes is given, and experimental confirmation 
of the enhanced resolving power for the one-dimensional coherent case is presented.

\section{COHERENT SUPERRESOLVING MICROSCOPE}

Neglecting magnification and, for simplicity, showing only one spatial dimension, we write the imaging equation for a coherent scanning microscope as

$$
\begin{aligned}
& i(y, \Delta x)=\int p_{\mathrm{c}}(-x) o(\Delta x-x) p_{\mathrm{o}}(y-x) \mathrm{d} x, \\
&-\infty<y<\infty,
\end{aligned}
$$

where $o(x)$ is the object amplitude, $p_{c}(x)$ is the form of the illumination, $p_{o}(y-x)$ is the amplitude point-spread function of the imaging lens, and $i(y, \Delta x)$ is the amplitude in the image plane when the object is centered at position $-\Delta x$. With the use of singular-system theory, ${ }^{6}$ an inversion of this equation yielding an estimate $o^{\prime}(x)$ for the form of the object is given by

$$
o^{\prime}(\Delta x-x)=\sum_{n=0}^{k} u_{n}(x) \frac{\int i(y, \Delta x) v_{n}(y) \mathrm{d} y}{\alpha_{n}},
$$

where $u_{n}(x)$ and $v_{n}(y)$ are the $n$th object and the imageplane singular functions, respectively, and $\alpha_{n}$ is the $n$th singular value. These singular functions and values are determined by the integral operator defined by Eq. (1). In the noise-free case the summation could, in principle, be taken to an infinite number of terms, and reconstruction would then contain frequencies of up to twice the cutoff of that of a conventional microscope and would have a more even distribution of frequencies than is obtained when a confocal scanning arrangement is used. In practice the summation must be finite; the value of $k$ is determined by the noise level in the measured image amplitude.

In general, for any scan position $\Delta x$, the inversion equation permits the reconstruction of a small region of the object amplitude that is centered around $\Delta x$. However, in a scanning system this is not required, it being sufficient at each scan position to obtain an estimate of the amplitude at the center of the illumination region and to use the scanning to build up the image. Hence at each scan position all that is required is an estimate for $o(\Delta x)$. Using Eq. (2), we can express this estimate in the form

$$
o^{\prime}(\Delta x)=\int i(y, \Delta x) h(y) \mathrm{d} y, \quad h(y)=\sum_{n=0}^{k} \frac{u_{n}(0) v_{n}(y)}{\alpha_{n}} .
$$

Thus it may be seen that the implementation of singularsystem processing in a scanning system involves the mathematically simple operation of multiplying the image complex amplitude $i(y, \Delta x)$ by a function $h(y)$ for each scanning position followed by spatial integration of the result. The function $h(y)$ is defined by the imaging operator and includes the effects of aberrations and polarizations. Despite the mathematical simplicity of this operation, it involves processing the complex amplitude of the imageplane light distribution. Hence, if a detector array is used to sample $i(y, \Delta x)$, not only do the individual detector ele- ments need to be accurately calibrated but the phase as well as the intensity at each element is required. This would necessitate that some form of phase-measuring interferometer be incorporated into the microscope, such that a reference beam also falls onto each element of the detector array. Clearly, the practical difficulties of implementing such an arrangement would be considerable.

However, the fact that both multiplication and spatial integration are operations that can be implemented optically presents an opportunity to carry out the processing of Eq. (3) optically before detection. An optical arrangement for implementing this superresolving processing in a coherent transmission-scanning microscope is shown in Fig. 1. The arrangement is similar to that in a confocal scanning microscope, with the image-plane pinhole replaced by a holographic element. The element has amplitude transmittance $h(y)$, which performs the multiplication part of the processing. The hologram is followed by a Fourier lens, which forms the Fourier transform of the premultiplied image-plane complex amplitude. The pinhole selects the zero-frequency component of the Fourier transform, which is equivalent to a spatial integration of the amplitude in the image plane. This combination of hologram, Fourier lens, and pinhole acts as a coherent optical processor, and a single detector placed behind this pinhole registers the intensity corresponding to the complex amplitude in Eq. (3). Thus, for a suitable form of $h(y)$ calculated by the singular system of the instrument or by other means, enhanced resolution is produced with the use of a single detector.

In general $h(y)$ is complex; thus the hologram has both amplitude and phase variations. This can be a composite hologram consisting of both a photographic plate that contains the intensity variations and an etched, or ion-milled, phase plate. A computer-controlled optical plotter can be used to perform both the exposure of the photographic plate and the exposure of the resist pattern that is used to control the etching, or ion-milling. However, in certain circumstances, a phase-only hologram can give results of sufficient quality that a composite hologram is not necessary.

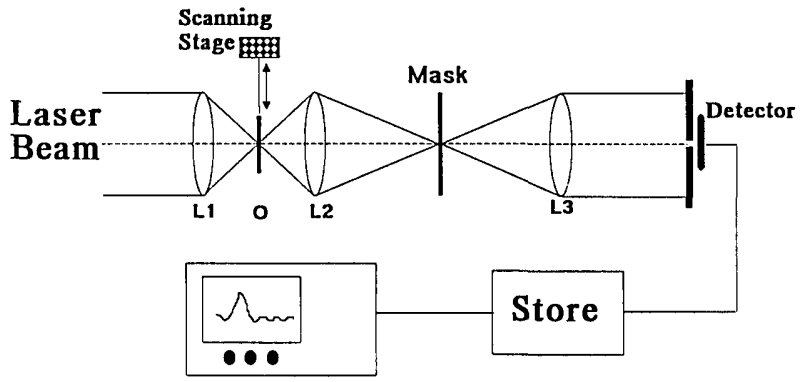

Fig. 1. Superresolving coherent microscope. Illuminating lens $\mathrm{L} 1$ focuses laser light onto small region of object $\mathrm{O}$. The object is scanned in two or three dimensions with respect to the focused light. An objective lens L2 images the illuminated region of the object onto a holographic mask, which multiplies the light amplitude by its complex amplitude transmittance. A lens L3 forms the Fourier transform of the amplitude distribution at the mask, and a pinhole selects the dc component. The operations of Fourier transformation and selection of the dc component are equivalent to a spatial integration of the complex amplitude in the plane of the mask and yield the superresolved signal to a detector behind the pinhole. Output from the detector is stored for subsequent display or analysis. 


\section{INCOHERENT SUPERRESOLVING MICROSCOPE}

Similarly to the coherent case, the one-dimensional imaging equation for an incoherent scanning microscope may be written as

$$
\begin{array}{r}
I(y, \Delta x)=\int P_{c}(-x) O(\Delta x-x) P_{o}(y-x) \mathrm{d} x, \\
-\infty<y<\infty,
\end{array}
$$

where $O(x)$ is the object intensity, $P_{\mathrm{c}}(x)$ is the form of the illumination intensity, $P_{o}(y-x)$ is the intensity pointspread function of the imaging lens, and $I(y, \Delta x)$ is the intensity in the image plane when the object is centered at position $-\Delta x$. When the singular-system theory is used, ${ }^{6}$ an inversion of this equation yielding an estimate, $O^{\prime}(x)$, for the form of the object intensity is given by

$$
O^{\prime}(\Delta x-x)=\sum_{n=0}^{k} U_{n}(x) \frac{\int I(y, \Delta x) V_{n}(y) \mathrm{d} y}{A_{n}},
$$

where $U_{n}(x)$ and $V_{n}(y)$ are the $n$th object and the imageplane singular functions, respectively, and $A_{n}$ is the $n$th singular value. These singular functions and values are determined by the integral operator that is defined by Eq. (4), and the value of $k$ is determined by the noise level in the measured image intensity.

As in the coherent case, it is sufficient at each scan position to obtain an estimate of the intensity at the center of the illumination region and to use the scanning to build up the image. With the use of Eq. (2), an estimate for $O(\Delta x)$ may be expressed in the form

$$
O^{\prime}(\Delta x)=\int I(y, \Delta x) H(y) \mathrm{d} y, \quad H(y)=\sum_{n=0}^{k} \frac{U_{n}(0) V_{n}(y)}{A_{n}} .
$$

Hence, as in the coherent case, the implementation of singular-system processing involves the mathematically simple operation of multiplying the image intensity by a function $H(y)$ followed by spatial integration of the result. Because the singular functions $U_{n}$ and $V_{n}$ are real, the function $H(y)$ is also real but will contain both positive and negative parts. Although in the incoherent case the processing involves intensities and no phase measurement is required, the necessity for a multielement detector array can be removed by partial optical processing with the use of the arrangement shown in Fig. 2. Light leaving the object is equally divided by a beam splitter to form two identical images on processing masks $\mathrm{M}+$ and $\mathrm{M}-$. These masks have different intensity transmittances; $\mathrm{M}+$ is denoted as the positive mask and $\mathrm{M}-$ as the negative mask. The positive mask has an intensity transmittance equal to $H(y)$ in those regions where $H(y)$ is positive; it has zero transmittance in the negative portions of $H(y)$. The negative mask has an intensity transmittance equal to $|H(y)|$ in those regions in which $H(y)$ is negative; it has zero transmittance elsewhere. The two large-area detectors integrate the positive and the negative signals, and these are then subtracted with an electronic subtractor to give a signal that is proportional to $O^{\prime}(\Delta x)$ in Eq. (6). As an alternative optical arrangement, the beam splitter and separate masks could be replaced by an optical element that is transmissive with transmittance $|H(y)|$ in those regions where $H(y)$ is positive and that is reflective with reflectance $|H(y)|$ in those regions where $H(y)$ is negative.

\section{FOURIER-OPTICS ANALYSIS OF IMAGING PROPERTIES}

In this section brief Fourier-optics analyses of the imaging properties of the novel microscopes are presented. For the coherent microscope, substituting Eq. (1) into Eq. (3) and rearranging gives

$$
o^{\prime}(\Delta x)=\int o(\Delta x-x) p_{c}(-x) \int p_{o}(y-x) h(y) \mathrm{d} y \mathrm{~d} x .
$$

With the symbol $\otimes$ used to denote convolution, the form of the amplitude at the detector may be expressed as

$$
o^{\prime}(\Delta x)=o(x) \otimes\left\{p_{c}(-x)\left[p_{o}(-x) \otimes h(x)\right]\right\} .
$$

Hence the amplitude point-spread function that is produced by this optical arrangement, $p_{s}(x)$, is given by

$$
p_{s}(x)=p_{c}(-x)\left[p_{o}(-x) \otimes h(x)\right],
$$

and the coherent transfer function, $\tilde{p}_{s}(f)$, is given by

$$
\tilde{p}_{s}(f)=\tilde{p}_{c}(-f) \otimes\left[\tilde{p}_{o}(-f) \tilde{h}(f)\right],
$$

where $\tilde{p}_{l}(f)$ and $\tilde{h}(f)$ are the Fourier transforms of $p_{l}(x)$ and $h(x)$, respectively.

For the incoherent microscope, substituting Eq. (6) into Eq. (4) and rearranging, we find the intensity point-spread function, $P_{s}(x)$, to be

$$
P_{s}(x)=P_{c}(-x)\left[P_{o}(-x) \otimes H(x)\right]
$$

and the incoherent transfer function, $\tilde{P}_{s}(f)$, to be

$$
\tilde{P}_{s}(f)=\tilde{P}_{c}(-f) \otimes\left[\tilde{P}_{o}(-f) \tilde{H}(f)\right],
$$

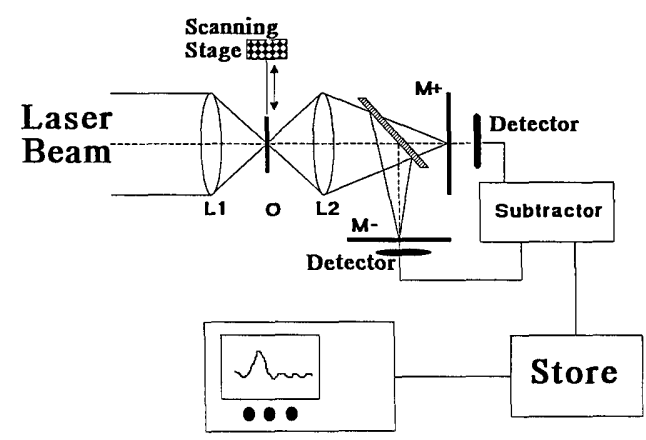

Fig. 2. Superresolving incoherent microscope. An illuminating lens L1 focuses laser light onto a small region of an object $\mathrm{O}$ stained with a fluorescent material. The object is scanned in two or three dimensions with respect to the focused light. Fluorescent light from the object is focused by an objective lens L2 and divided by a beam splitter to form two identical incoherent images on two intensity masks $\mathrm{M}+$ and $\mathrm{M}-$. The intensity mask $\mathrm{M}+$ performs the processing that corresponds to the positive portions of the complete mask function (the negative portions being opaque), and the mask $\mathrm{M}$ - performs the processing that corresponds to the modulus of the negative portions of the mask (the positive portions being opaque). A signal obtained from an integrating detector behind mask $\mathrm{M}$ - is electronically subtracted from that of mask $M+$ to produce a superresolved intensity signal, which is stored for subsequent display or analysis. 
where $\tilde{P}_{l}(f)$ and $\tilde{H}(f)$ are the Fourier transforms of $P_{l}(x)$ and $H(x)$, respectively.

The results in Eqs. (9)-(12) reduce to the known results for confocal microscopy if appropriate mask functions are used. In conventional confocal microscopy the mask positions in Figs. 1 and 2 are occupied by pinhole apertures. This corresponds to the fact that the mask functions, $h(x)$ and $H(x)$, are Dirac delta functions. In this situation Eqs. (9) and (11) reduce to the known result [see, e.g., Eq. (3.35) of Ref. 1] that the point-spread function is the product of the point-spread functions of the two lenses. In the Fourier domain, for pinhole apertures, $\tilde{h}(f)$ and $\tilde{H}(f)$ become constant functions, and the transfer functions [Eqs. (10) and (12)] reduce to the known result that the confocal transfer function is the convolution of the transfer functions of the two lenses.

The enhanced resolving power of the point-spread function given by Eq. (9) is now shown by a simple example. Consider the case of two identical aberration-free onedimensional lenses of numerical aperture $N$; then

$$
p_{c}(x)=p_{o}(x)=\operatorname{sinc}\left(\frac{2 \pi N x}{\lambda}\right), \quad \operatorname{sinc}(\theta)=\frac{\sin (\theta)}{\theta} .
$$

In this one-dimensional coherent case it is not difficult, with a simple Fourier argument, to derive the optimum form for the mask function. This is achieved by defining the desired transfer function as that which uniformly fills twice the conventional microscope band and by using simple Fourier-optics theory. This approach yields the optimum mask function,

$$
h(x)=\cos \left(\frac{2 \pi N x}{\lambda}\right)
$$

Using this form of $h(x)$, we can give the effective pointspread function by

$$
\begin{aligned}
p_{s}(x) & =\operatorname{sinc}\left(\frac{2 \pi N x}{\lambda}\right)\left[\operatorname{sinc}\left(\frac{2 \pi N x}{\lambda}\right) \otimes \cos \left(\frac{2 \pi N x}{\lambda}\right)\right] \\
& =\operatorname{sinc}\left(\frac{4 \pi N x}{\lambda}\right) .
\end{aligned}
$$

This point-spread function gives better resolution (in terms of reproducing the higher object spatial frequencies with more power) than either a conventional microscope with a point-spread function of $\operatorname{sinc}[(2 \pi N x) / \lambda]$ or a conventional scanning confocal microscope with a pointspread function of $\operatorname{sinc}^{2}[(2 \pi N x) / \lambda]$. Note, in particular, that the first zeros of the point-spread function in Eq. (15) are spaced at half the distance of the first zeros in the point-spread functions of both the conventional microscope and the confocal microscope. The transfer function associated with this point-spread function has a top-hat form that is twice as wide as that of a conventional microscope; thus the effect of the optical arrangement in Fig. 1 is to perform as a conventional coherent microscope with lenses of doubled numerical aperture.

In this one-dimensional coherent case, we can use a phase-only hologram instead of the cosine form of Eq. (14) to achieve the same resultant point-spread function. The phase-only hologram that accomplishes this point-spread function has the form $h_{\phi}(x)=\operatorname{sgn}\{\cos [(2 \pi N x) / \lambda]\}$. We can easily see that this hologram has the same effect if we note that, in Eq. (10), $\tilde{h}(f)$ is multiplied by $\tilde{p}_{o}(-f)$; that $\tilde{p}_{o}(f)$ has a limit of $N / \lambda$; and that $h_{\phi}(x)$ is identical to the cosine form of $h(x)$ within the band of $\tilde{p}_{o}(f)$. The ability to achieve the enhanced resolution with a phase-only mask has important practical implications, because accurate phase structures are normally much easier to fabricate than accurate amplitude structures. The results of using such a phase structure are presented in Section 5 .

\section{EXPERIMENTAL RESULTS}

To demonstrate the performance of this approach to superresolution, a coherent, one-dimensional low-numerical-aperture transmission microscope similar to that illustrated in Fig. 1 was used. Lenses L1 and L2 had a focal length of $20 \mathrm{~cm}$, and light of wavelength $0.488 \mu \mathrm{m}$ was used. Object $O$ was a vertical clear line of width $R / 20$, where $R$, the Rayleigh distance, defined by the use of slit apertures in the lens Fourier planes, was approximately $360 \mu \mathrm{m}$. A mask with a phase-only weighting was used. This mask was produced by using a computer-controlled optical plotter to write a Ronchi grating with a period equal to twice the Rayleigh distance in a layer of photoresist on a glass plate. The plate was etched to a depth of $\lambda / 2(n-1)$, where $n$ is the refractive index of the glass plate, and the remaining photoresist was removed.

Results are presented in Fig. 3. The point-spread function of the microscope was measured by scanning the narrow slit object both with the mask removed and with the mask in place. The circles show the measured pointspread function obtained with the mask removed; this point-spread function is equivalent to that of a conventional coherent microscope of the same numerical aperture. The diamonds show the point-spread function data obtained with the mask in position. The increase in resolution over that obtained with the conventional microscope is evident. Note in particular that the position of the first zero of the point-spread function lies close to half that of the conventional data. This may be compared with the point-spread function of a scanning confocal microscope, which is sharper than that of a conventional microscope but has zeros at the same positions. This point-spread function shows a gain in resolution by approximately a

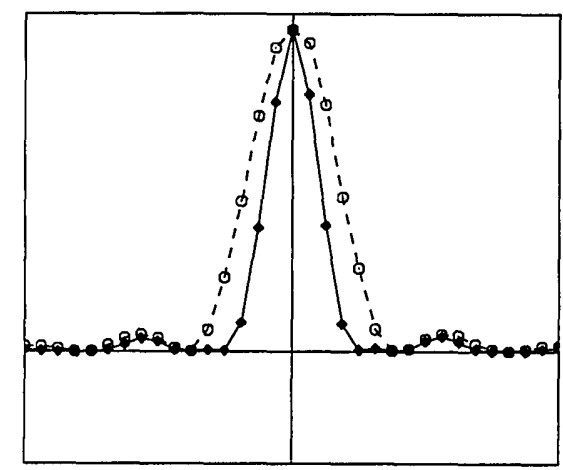

Fig. 3. Experimental results. The circles show the measured point-spread function obtained with the mask removed; this point-spread function is equivalent to that of a conventional coherent microscope of the same numerical aperture. The diamonds show the point-spread function data obtained with the mask in position. The increase in resolution over the conventional microscope, by approximately a factor of $1.7-1.8$, is evident. 
factor of 1.7-1.8 over the conventional microscope. With further experimental work, it is expected that these results can be improved, with a gain in resolution over the conventional system of greater than 1.9 .

\section{DISCUSSION}

The Fourier analysis, used in Section 4 for the one-dimensional coherent case, is also useful in the two-dimensional coherent case for square or rectangular apertures. However, because of division-by-zero problems, such an approach is not possible for the experimentally important case of circular lens apertures. For the same reason, simple Fourier techniques may not be applied to the incoherent case for any shape of aperture. In these cases, the forms of the holographic masks are calculated with a singular-system analysis. Such calculations may include the effects of known lens aberrations in both the illumination and the imaging systems. The effects of aberrations on the performance of such a microscope have been studied $^{15}$; further details will be published later, but the method is found to be robust.

In general, the two-dimensional coherent holographic masks that we have calculated will have both amplitude and phase variations. The effect of using phase-only versions of these masks is under investigation; preliminary results suggest that phase-only masks give less resolution enhancement than masks with both phase and amplitude variations but that substantial enhancement is still obtained. The intensity masks, calculated for the incoherent microscope, have continuously variable intensity transmittance. The performance of binary masks, which have only two intensity transmittances and which are far simpler to fabricate, is also under investigation. We plan to publish detailed results of these studies.

For the coherent case, the same lateral resolution enhancement may be achieved with a confocal arrangement that has a mask placed in the imaging-lens pupil plane with a transfer function of form $\tilde{h}(f)$. This effect may be seen if we recall that for a coherent system the transfer function of a lens is a scaled version of the pupil function. Thus, for a mask with transfer function $\tilde{h}(f)$ placed in the pupil plane, the combined transfer function is $\tilde{h}(f) \tilde{p}_{o}(f)$. In confocal microscopy the transfer function is the convolution of the transfer functions of the two lenses. Thus the use of an imaging-lens pupil-plane mask of suitable form gives the same resultant transfer function as given by Eq. (10). In this way singular-system theory may be regarded as a means of designing an optimum imaginglens pupil function. The use of annular apertures to achieve a limited degree of resolution enhancement has been discussed previously (see, e.g., page 49 of Ref. 1). Practical considerations will determine whether an imageplane mask used with a Fourier lens and a pinhole or a pupil-plane mask used in a confocal arrangement is preferable. One important consideration is that an imageplane mask could be a phase-only structure, whereas a pupil-plane mask would usually require both phase and amplitude variations.

The microscope's property of being able to use a mask in the image plane, as shown in Fig. 1, or its scaled Fourier transform in the pupil plane does not apply to the incoherent case. In an incoherent system the transfer functions are related to the autocorrelation of the pupil functions, and no simple relation between the intensities in the pupil and the image planes exists. It should also be noted that the above argument applies only to the lateral resolution of planar objects.

\section{CONCLUSIONS}

Two novel superresolving scanning microscopes are described; these instruments have been patented. ${ }^{14}$ The coherent arrangement allows singular-system processing to be implemented with a single detector and without the need for direct phase measurement. The incoherent microscope performs the processing with two detectors and an electronic subtractor. A simple Fourier analysis is presented that gives expressions for the point-spread and transfer functions in terms of the mask functions. The enhancement in resolving power for the one-dimensional coherent case is shown to be a factor of 2 better than for a conventional microscope. In this simple case it is shown that a phase-only mask has the same effect as a phase and amplitude mask. In all but a few cases, singular-system analysis is the preferable method for calculating the mask functions. Preliminary results for the one-dimensional case have demonstrated the practical feasibility of this approach to resolution enhancement.

Future work will assess the performance of twodimensional phase-only masks for the coherent case and of binary-intensity masks for the incoherent case.

${ }^{*} \mathrm{M}$. R. Young is currently at the University of Arkansas, Fayetteville, Arkansas 72701.

\section{REFERENCES}

1. T. Wilson and C. J. R. Sheppard, Theory and Practice of Scanning Optical Microscopy (Academic, New York, 1984).

2. G. J. Brakenhoff, P. Blom, and P. Barends, "Confocal scanning light microscope with high aperture lenses," J. Microsc. (Oxford) 117, 219-232 (1978).

3. M. Bertero and E. R. Pike, "Resolution in diffraction-limited imaging, a singular value analysis: I. The case of coherent illumination," Opt. Acta 29, 727-746 (1982).

4. M. Bertero, P. Boccacci, and E. R. Pike, "Resolution in diffraction-limited imaging, a singular value analysis: II. The case of incoherent illumination," Opt. Acta 29, 1599-1611 (1982).

5. M. Bertero, P. Brianzi, P. Parker, and E. R. Pike, "Resolution in diffraction-limited imaging, a singular value analysis: III. The effect of sampling and truncation of the data," Opt. Acta 31, 181-201 (1984).

6. M. Bertero, C. De Mol, E. R. Pike, and J. G. Walker, "Resolution in diffraction-limited imaging, a singular value analysis: IV. The case of uncertain localisation of nonuniform illumination of the object," Opt. Acta 31, 923-946 (1984).

7. D. Slepian and H. O. Pollak, "Prolate spheroidal wave functions, Fourier analysis and uncertainty," Bell Syst. Tech. J. 40, 43-63 (1961).

8. J. G. Walker, "Optical imaging with resolution exceeding the Rayleigh criterion," Opt. Acta 30, 1197-1202 (1983).

9. M. Bertero, P. Brianzi, and E. R. Pike, "Superresolution in confocal scanning microscopy," Inverse Probl. 3, 195-212 (1987).

10. M. Bertero, C. De Mol, and E. R. Pike, "Analytic inversion formula for confocal scanning microscopy," J. Opt. Soc. Am. A 4, 1748-1750 (1987).

11. M. R. Young, R. E. Davies, E. R. Pike, and J. G. Walker, "Superresolution in confocal scanning microscopy: confirmation in the 1-d incoherent case," in Digest of Conference on 
Signal Recovery and Synthesis 3 (Optical Society of America, Washington, D.C., 1989), paper WD4.

12. M. R. Young, R. E. Davies, E. R. Pike, and J. G. Walker, "Superresolution in confocal scanning microscopy: confirmation in the 1-d coherent case," Europhys. Lett. 9, 773-778 (1989).

13. E. R. Pike, R. E. Davies, J. G. Walker, and M. R. Young, "An introduction to singular systems with applications to confocal scanning microscopy," J. Microsc. (Oxford) 160, 107-114 (1990).

14. J. G. Walker, E. R. Pike, and M. Bertero, "Scanning optical microscope," British Patent Application 89/13129 (September 1989).

15. R. E. Davies, "Inverse problems in confocal scanning microscopy," Ph.D. dissertation (University of London, London, 1990). 